\title{
Lista actualizada de la flora marina bentónica de cinco arrecifes coralinos del norte de Veracruz, México
}

\section{Updated list of marine benthic flora from five coral reefs in northern Veracruz, Mexico}

\author{
Vicencio de la Cruz-Francisco ${ }^{1}$, Rosa Estela Orduña-Medrano ${ }^{1}$ y \\ Marlene González-González ${ }^{1}$
}

\section{RESUMEN}

El macrofitobentos marino cumple un papel importante en los arrecifes coralinos, sin embargo, son de los grupos biológicos menos estudiados en el Sistema Arrecifal Lobos-Tuxpan, Veracruz, México (SALT). El objetivo del presente estudio fue actualizar la lista taxonómica de las especies de algas y fanerógamas presentes en los arrecifes Tuxpan, Enmedio, Tanhuijo, Lobos y Oro Verde. Entre el periodo 2014-2018, se realizaron un total de 38 muestreos, las exploraciones fueron en la laguna arrecifal y en las pendientes profundas de los arrecifes mediante buceos errantes. La búsqueda de algas y fanerógamas marinas se realizó en cinco tipos de sustratos: arena, guijarros, rocas coralinas, pastos marinos y zona de corales vivos. Así mismo, el listado taxonómico se complementó con registros de especies reportadas previamente para el SALT. La lista taxonómica actualizada está compuesta por 130 especies: 2 pertenecen a Cyanobacteria, 55 Rhodophyta, 23 Ochrophyta, 48 Chlorophyta y 2 Tracheophyta representadas por Halophila decipiens y Thalassia testudinum. Del total de especies, 28 taxa se citan por primera vez para la zona de estudio. Además, Sphorochnus pedunculatus constituye un nuevo registro para el suroeste del golfo de México. Los sustratos con mayor riqueza de algas fueron rocas coralinas y guijarros. Los arrecifes emergentes Lobos, Enmedio y Tuxpan presentaron mayor riqueza florística. La presente información representa un buen avance en el conocimiento de la flora marina y puede ser de utilidad para la toma de decisiones eficaces en materia de manejo, en especial para las fanerógamas que presentan un estatus de riesgo.

Palabras clave: algas, arrecifes, fanerógamas, fitobentos, sustratos

\begin{abstract}
Marine macrophytobenthos plays an important role in coral reefs; however, it is one of the least studied biological groups in the Lobos-Tuxpan Reef System (SALT) in Veracruz, Mexico. The objective of the present study was to update the taxonomic list of the algae and phanerogam species present in the Tuxpan, Enmedio, Tanhuijo, Lobos, and Oro Verde reefs. During the 2014-2018 period, 38 surveys were conducted in the reef lagoon and the deep slopes of the

1 Facultad de Ciencias Biológicas y Agropecuarias, Campus Tuxpan, Universidad Veracruzana. Carr. Tuxpan-Tampico km 7.5, Col. Universitaria CP. 92860, Tuxpan, Veracruz, México. viccruz@uv.mx*. ORCID: orcid.org/00000001-8339-6730; ORCID: orcid.org/0000-0003-2458-6221; ORCID: orcid.org/0000-0002-2095-8457
\end{abstract}


reefs by errant dives. Algae and marine phanerogams were surveyed on five types of substrates: sand, pebbles, coral rocks, seagrasses, and live coral area. In addition, the taxonomic list was complemented with records of species previously reported for SALT. The updated taxonomic list includes 130 species: 2 being Cyanobacteria, 55 Rhodophyta, 23 Ochrophyta, 48 Chlorophyta, and 2 Tracheophyta, represented by Halophila decipiens and Thalassia testudinum. Of the total species, 28 taxa are cited for the first time for the study area. Additionally, Sphorochnus pedunculatus constitutes a new record for the southwest of the Gulf of Mexico. The substrates with the highest algae richness were coral rocks and pebbles. The Lobos, Enmedio, and Tuxpan emerging reefs presented greater floristic wealth. This information represents a good advance in the knowledge of marine flora and can be useful for effective management decision-making, especially for phanerogams, which are in a risk status.

Keywords: algae, reefs, phanerogams, phytobenthos, substrates

\section{INTRODUCCIÓN}

El macrofitobentos marino incluye a las algas y pastos marinos, ambos grupos son importantes en los ecosistemas arrecifales dado que forman parte del componente bentónico de los arrecifes de coral. Las algas arrecifales manifiestan una gran diversidad de especies, son abundantes y contribuyen en la productividad primaria, además son competidores por el espacio con los corales, y sus frondas proveen refugio y alimento para la fauna marina (Fong \& Paul, 2011; Ramírez-Rodríguez \& Blanco-Pérez, 2011; Aguilera-Pérez \& De la Cruz-Francisco, 2017). Por igual, los pastos marinos o fanerógamas desempeñan un papel importante en los arrecifes dado son productores primarios, participan en el reciclamiento de nutrientes, atenúan el oleaje, retienen el sedimento, sus praderas sirven de crianza, alimento y reproducción para la fauna marina, y constituyen un excelente sustrato para la fijación de epifitas (Romeu, 1996; Ramírez-García et al. 2007; Cervantes-Maldonado \& Quintero, 2016).

En los arrecifes coralinos de Veracruz, la comunidad de algas está constituida principalmente por algas rojas, los géneros más comunes son Acanthophora, Amphiroa, Ceramium, Galaxaura, Gracilaria, Hypnea, Jania y Laurencia, de igual manera, la abundancia de algas verdes es importante y representada por los géneros Caulerpa, Cladophora y Ulva, también, las algas pardas son predominantes en los arrecifes esencialmente por los géneros Dictyota y Padina (Lehman, 2010; Ramírez-Rodríguez \& Blanco-Pérez, 2011). Respecto a los pastos marinos, dos especies se han reportado en los arrecifes veracruzanos: Syringodium filiforme y Thalassia testudinum, esta última fanerógama es la más abundante en cobertura y biomasa, sobre todo se establece en la laguna arrecifal (Godínez-Ortega et al. 2015). 
Si bien, Veracruz es la localidad más explorada y con mayor diversidad ficológica para las costas mexicanas del golfo de México (Ortega et al. 2001; Pedroche \& Sentíes, 2003), aún se considera que es limitado el conocimiento de la riqueza florística ligada a los arrecifes veracruzanos a pesar de los esfuerzos de investigación realizados principalmente en el Sistema Arrecifal Veracruzano (SAV) (Galicia-García \& Morales-García, 2007; Robinson et al. 2012; Galicia-García et al. 2013; Godínez-Ortega et al. 2015; 2019), además, se enfatiza que el ambiente submareal ha sido poco explorado (Ramírez-Rodríguez \& Blanco-Pérez, 2011; Godínez-Ortega et al. 2019). Respecto a los pastos marinos, también han sido objeto de estudio, a la fecha se tiene conocimiento de su distribución, abundancia, biomasa, productividad y crecimiento (Lot-Helgueras, 1971; Ibarra-Morales \& Abarca-Arenas, 2007; Ramírez-García et al. 2007; Arellano-Méndez et al. 2016), así como su estructura comunitaria (Godínez-Ortega et al. 2015).

De forma inversa, el Sistema Arrecifal Los Tuxtlas (SAT) carece de inventarios ficológicos, este sistema se distribuye al sur de Veracruz y apenas se están haciendo esfuerzos por determinar su biodiversidad. Respecto al Sistema Arrecifal Lobos-Tuxpan (SALT), ubicado al norte de Veracruz, son pocas las investigaciones que han explorado la flora marina donde la laguna arrecifal, ha sido la zona arrecifal más estudia. Entre la década de los sesenta y principios de los setenta, se realizaron los primeros estudios de la flora marina en el SALT, inicialmente, Barrientos (1965) aporta un listado preliminar de las algas del arrecife Blanquilla. Huerta-Múzquiz \& Barrientos (1965) contribuyen con los primeros registros de algas para el arrecife Lobos, y más tarde, Chávez et al. (1970) incrementan el conocimiento florístico con datos de la composición y distribución de las algas y del pasto marino Thalassia testudinum, información que a la fecha no ha sido actualizada. Posteriormente, a principios de la década del 2000, varios estudios realizados en el arrecife Tuxpan determinaron la composición y distribución de las algas en diferentes hábitats arrecifales (Ortega et al. 2001; González-Gándara et al. 2007; Aguilera-Pérez \& De la Cruz-Francisco, 2017). Mientras, para los arrecifes Enmedio y Oro Verde se tiene poco conocimiento de la flora marina, dado que se han registrado menos de 25 especies de algas para estos arrecifes (González-González et al. 2016; Luna-Ortega \& De la Cruz-Francisco, 2017).

Si bien, todos estudios han enriquecido el conocimiento de la flora marina para el SALT, la realización de más estudios y exploraciones son necesarias para incrementar el conocimiento sobre la riqueza del macrofitobentos en lugares arrecifales menos conocidos, como las pendientes profundas 
(Pedroche \& Sentíes, 2003; Galicia-García \& Morales-García, 2007). Por ello, el propósito del presente trabajo fue proporcionar un listado actualizado de la flora marina bentónica, basado en recolectas y observaciones hechas desde el 2014 al 2018 en cinco de los diez arrecifes del SALT, así como la integración de registros previos de la literatura existente. Dado que el SALT es área natural protegida, la información presentada en este trabajo puede servir de apoyo para los planes de manejo y conservación a fin de proteger aquellas especies que presentan un estatus de riesgo con base en la NOM-059-SEMARNAT-2010 (DOF, 2019).

\section{MATERIALES Y METODOS}

Área de estudio. Al norte del estado de Veracruz, en el golfo de México (Fig. 1) se localiza el Área Natural Protegida Sistema Arrecifal Lobos-Tuxpan (SALT), decretado en el año 2009 como Área de Protección de Flora y Fauna de México (DOF, 2009). Está integrado por dos polígonos de protección; el polígono Lobos lo componen tres arrecifes coralinos emergentes: Lobos, Medio y Blanquilla, mientras el polígono Tuxpan lo conforman tres arrecifes emergentes: Tanhuijo, Enmedio, Tuxpan y el arrecife sumergido Pantepec (Ortiz-Lozano et al. 2013). En las inmediaciones de estos polígonos se han cartografiado otros arrecifes sumergidos nombrados como arrecife Oro Verde, arrecife Pantepec Sur y arrecife Corazones (Ortiz-Lozano et al. 2019) (Fig. 1). Este complejo arrecifal forma parte del Corredor Arrecifal del suroeste del golfo de México (Ortiz-Lozano et al. 2019) (Fig. 1).

Para elaborar la lista taxonómica del macrofitobentos, se efectuaron un total de 38 salidas a los arrecifes del SALT durante el 2014-2018. Se dedicó un tiempo de trabajo en cada salida de 4 h/día. Para el arrecife Enmedio, se realizaron al menos 12 muestreos (equivalente a $46 \mathrm{~h}$ ) que procedieron de estudios que determinaron la distribución y cobertura bentónica de dicho arrecife (De la Cruz-Francisco et al. 2016; González-González et al. 2016) durante abril-noviembre 2015 y abril-mayo 2016, donde gran parte del material biológico recolectado quedó pendiente por identificar, posteriormente se efectuaron dos salidas más en abril 2017 (8 h) y abril de 2018 (8 h). Mientras, para el arrecife Tuxpan se efectuaron seis muestreos $(24 \mathrm{~h})$ en los meses de mayo y julio 2014; abril 2015; mayo 2016 y 2017, así también, seis exploraciones $(24 \mathrm{~h})$ se efectuaron para el arrecife Tanhuijo (noviembre 2014; julio-agosto 2015) y arrecife Oro Verde (junio-agosto 2015) respectivamente. Respecto al arrecife Lobos, se realizó un muestreo en mayo 2014 $(4 \mathrm{~h})$, después en noviembre 2016 (4 h), y por último, una estancia de cinco días en noviembre de 2017 (20 h). 

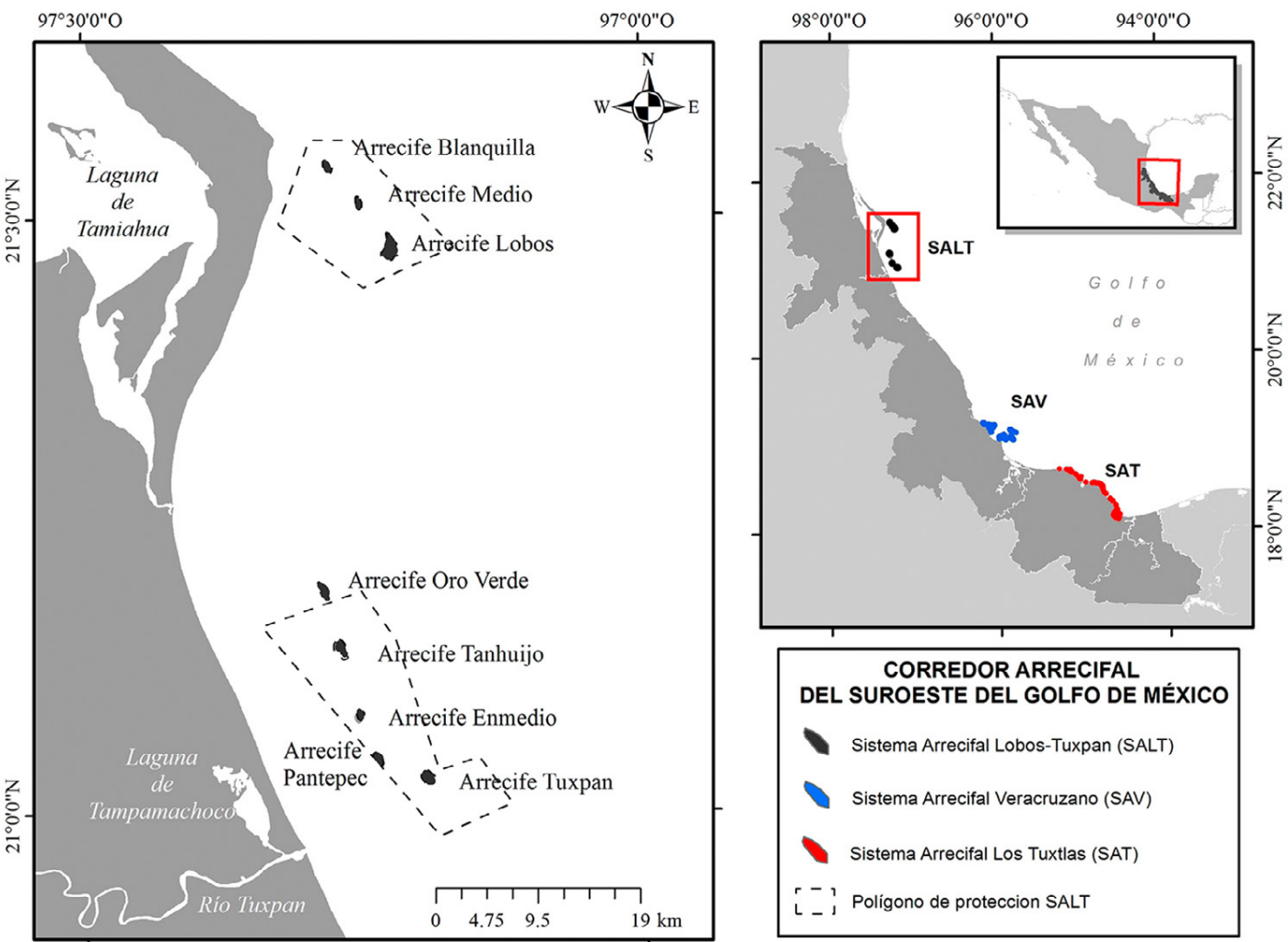

Fig. 1. Localización geográfica de los arrecifes coralinos que conforman el Sistema Arrecifal Lobos-Tuxpan (SALT)

Fig. 1. Geographical location of the coral reefs that make up the Lobos-Tuxpan Reef System (SALT, its acronym in Spanish)

Los muestreos consistieron en la búsqueda de algas y fanerógamas marinas en cinco tipos de sustratos, en especial a) parches de arena, b) guijarros, c) rocas, d) y en zonas de pastos marinos (Thalassia testudinum L.) y de e) corales vivos. Para esto, se aplicó la técnica del buzo errante, el cual consistió en nadar libremente durante 20-30 minutos en cada uno de los sustratos establecidos para la identificación y recolecta de especímenes dudosos. En cada ambiente se tomaron datos de los ejemplares observados (profundidad, sustrato, localidad, coordenadas, fecha, colector, así como datos de color, forma y textura) y posteriormente se recolectaron (solo los necesarios para la identificación) desprendiéndolas desde la base del sustrato con una navaja para el caso de las macroalgas corticadas, foliosas y sifonales, mientras para los tapetes algales se utilizó una espátula y se 
recolectaron alrededor de dos muestras de $20 \mathrm{~cm}^{2}$ por muestreo (Flores-Leiva et al. 2010). Las muestras se colocaron en bolsas de polietileno y fueron etiquetadas, luego se almacenaron en neveras de unicel para protegerlas de la radiación solar (Flores-Leiva et al. 2010). Cabe mencionar, que para la laguna arrecifal, los muestreos se realizaron con equipo de buceo libre, mientras, para las zonas profundas se efectuaron inmersiones de $2-20 \mathrm{~m}$ de profundidad con equipo de buceo autónomo. En el laboratorio de la Facultad de Ciencias Biológicas de la Universidad Veracruzana, las muestras biológicas se preservaron en líquido, mediante una solución de formalina al 4\% (Flores-Leiva et al. 2010) y después se depositaron en la colección biológica de la propia facultad.

Para la identificación de las algas, primero se realizaron cortes transversales de los talos con navajas de afeitar (marca Gillette) y un microscopio estereoscópico (marca Nikon SMZ745), posteriormente se prepararon laminillas temporales (usando portaobjetos y cubreobjetos) para observar las estructuras reproductoras y vegetativas en un microscopio óptico (marca Nikon modelo Eclipse E-100). También, se tuvieron en cuenta especies epifitas en macroalgas frondosas (e. g. Galaxaura, Laurencia, Padina, Dictyota, Rhipocephalus, Halimeda). Para los ejemplares con estructura calcárea de las familias Corallinacea,
Dichotomosiphonaceae, Udoteaceae, y Dasycladaceae, se utilizó ácido clorhídrico al $15 \%$ para la descalcificación de los tejidos (Aguilar-Rosas et al. 2010). La determinación del material algal se llevó a cabo mediante guías de identificación (Littler \& Littler, 2000; León-Álvarez et al. 2007) y trabajos especializados (Sentíes \& Fujii, 2002; Solé \& Foldats, 2003; Bandeira-Pedrosa et al. 2004; Alves et al. 2010; Pacheco-Cervera et al. 2010; Robinson et al. 2012; Galicia-García et al. 2013; Mendoza-González et al. 2014; Moura et al. 2014; Vieira et al. 2016; Godínez-Ortega et al. 2019). Para el reconocimiento de las fanerógamas marinas se utilizaron los trabajos de van Tussenbroek et al. (2010) y Martínez-Daranas et al. (2013).

Para complementar el listado taxonómico, se adicionaron los registros de algas reportados para el SALT con base en los trabajos de Huerta-Múzquiz \& Barrientos (1965), Chávez et al. (1970), Ortega et al. (2001), GonzálezGándara et al. (2007), GonzálezGonzález et al. (2016), Aguilera-Pérez \& De la Cruz-Francisco (2017) y Luna-Ortega \& De la Cruz-Francisco (2017). Con la información obtenida, se elaboró un listado sistemático que sigue al criterio de Zúñiga-Ríos et al. (2012) para las categorías taxonómicas superiores: División y Orden, mientras, las especies dentro de cada orden se establecieron alfabéticamente. Además, la nomenclatura de los nombres 
científicos se actualizó al consultar la base de datos Algaebase (Guiry \& Guiry, 2019).

\section{RESULTADOS}

En el presente estudio se identificaron en total 92 especies de algas y dos especies de fanerógamas en el sistema arrecifal SALT. Se complementaron estos resultados con las 102 especies que han sido reportadas, con anteriori$\mathrm{dad}$, en la literatura, el inventario taxonómico de la flora marina bentónica para esta zona queda integrada por 130 especies, repartidas en cinco divisiones y dos reinos (Cuadro 1). Rhodophyta (algas rojas) y Chlorophyta (algas verdes) presentaron mayor número de taxones con 55 y 48 especies respectivamente. Las familias con mayor riqueza fueron: Dictyotaceae con 16 especies, seguido de Rhodomelaceae con 10 especies y Caulerpaceae con nueve especies (Cuadro 2). Del total de la riqueza, 28 especies encontradas en este estudio constituyen los primeros registros para el área natural protegida, en su mayoría corresponden a Chlorophyta (Cuadro 1 y 2). Adicionalmente, se amplía la distribución de Sporochnus pedunculatus para el suroeste del golfo de México, su descripción morfológica se muestra al final de esta sección.
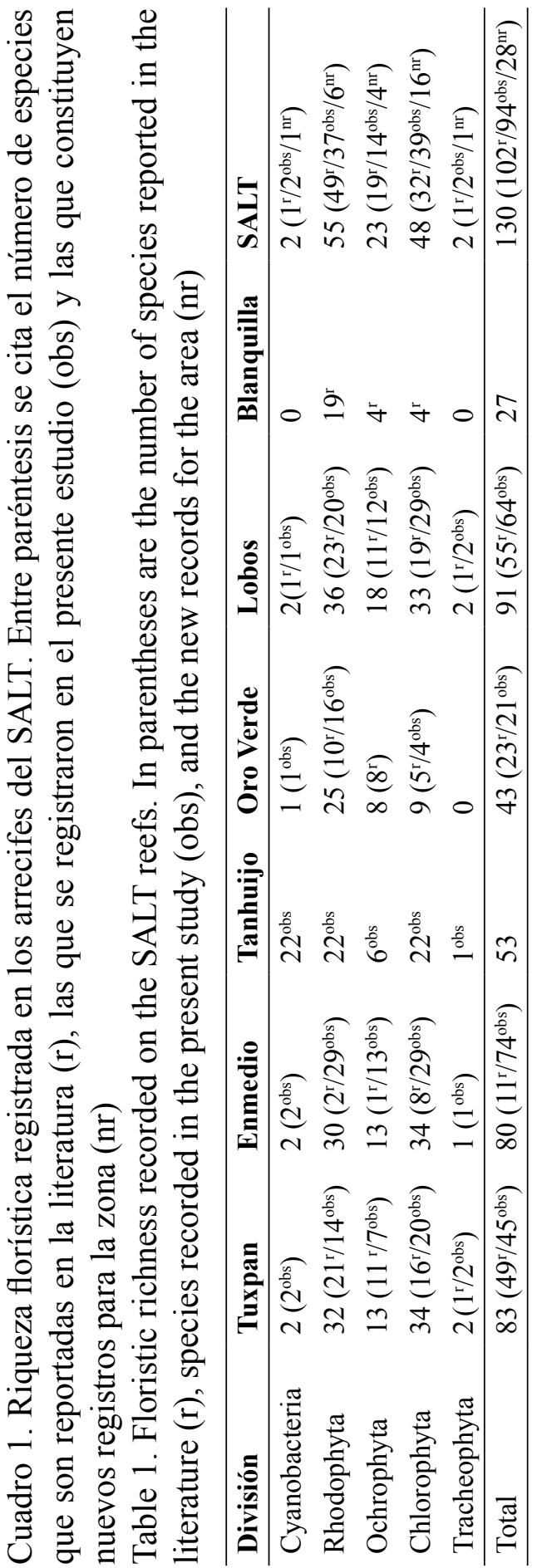


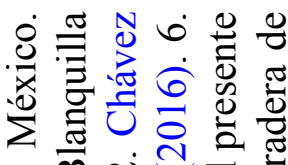

舟



ज.

:

年.

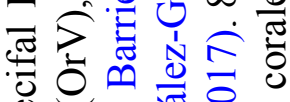

政

踏

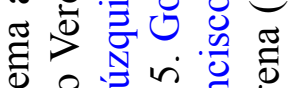

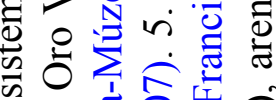

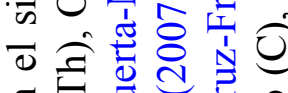

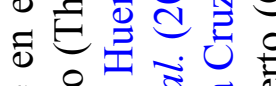

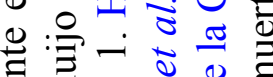

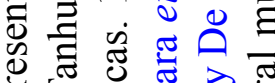

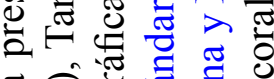

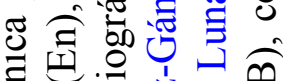

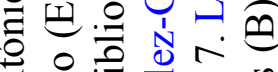

部啳

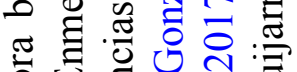



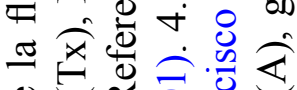

\%



娄守





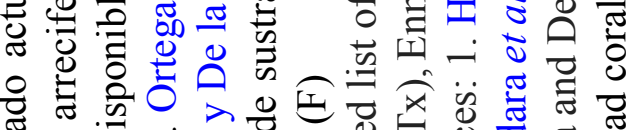

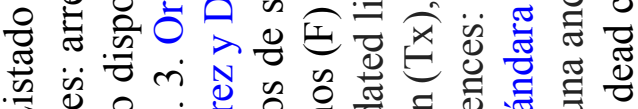

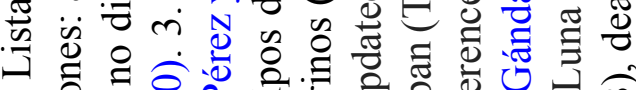

人응

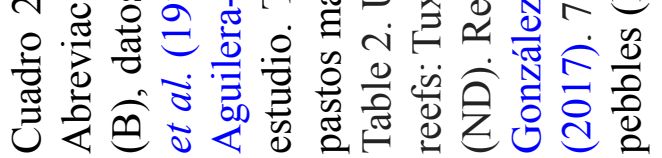

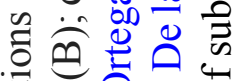

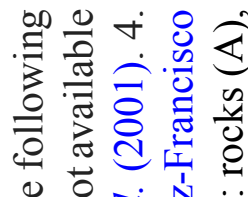

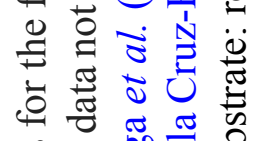

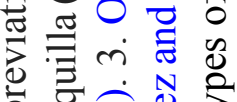



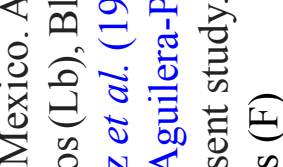

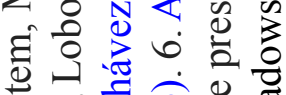

茫它它 导

๘

ข

हี่

O

ᄋ

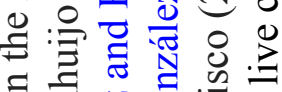



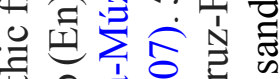

음의 항



\& $\sim$

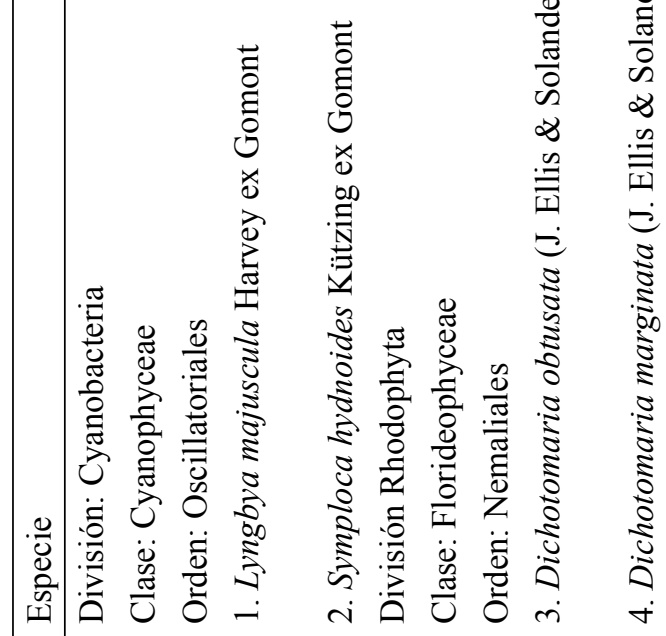

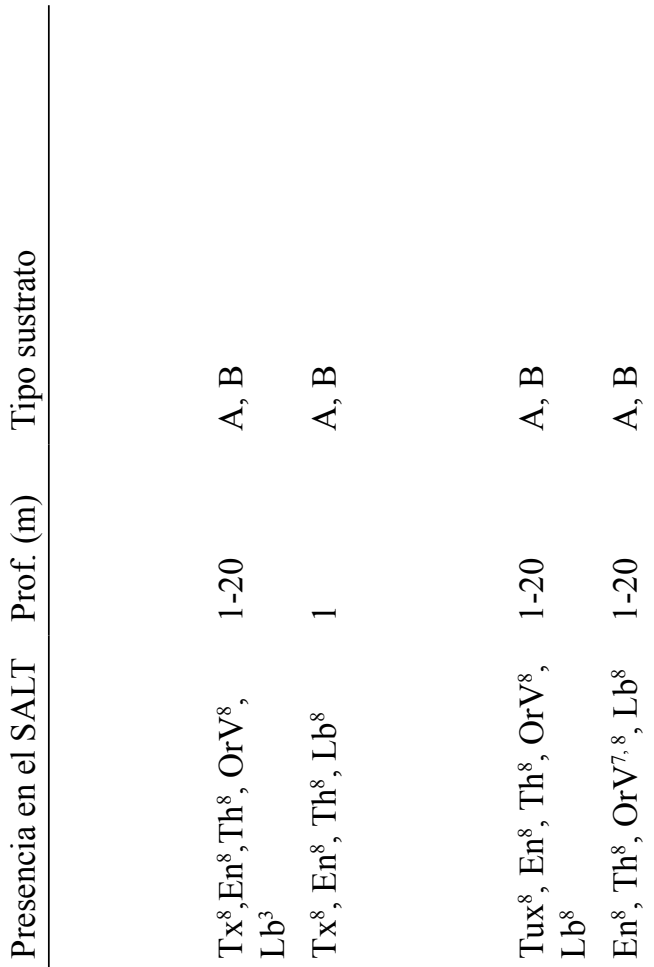




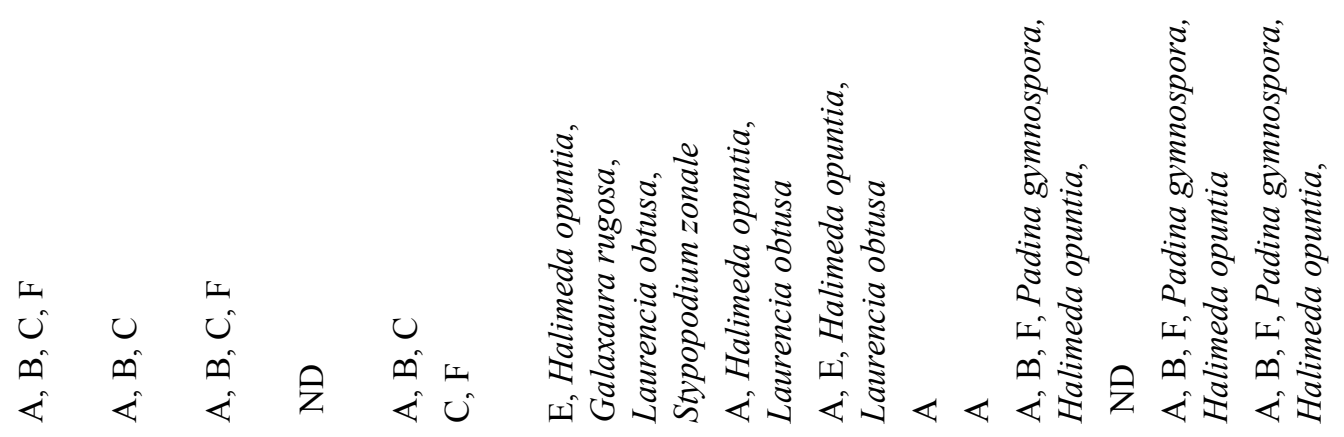

จ
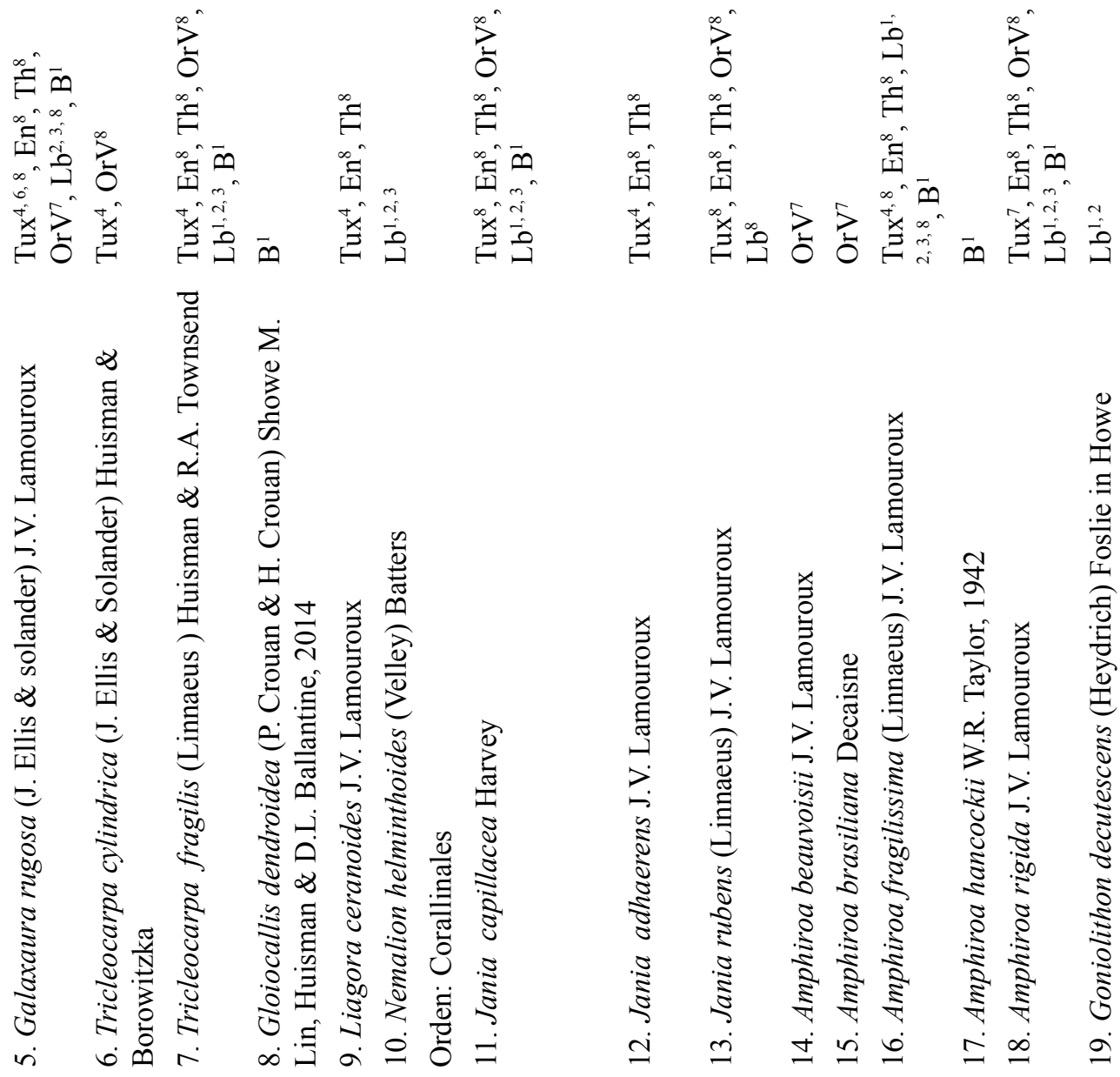


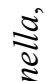

के

$\stackrel{2}{8}$

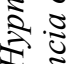

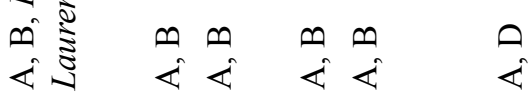

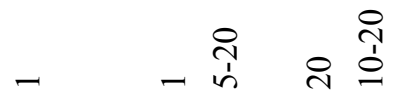

两

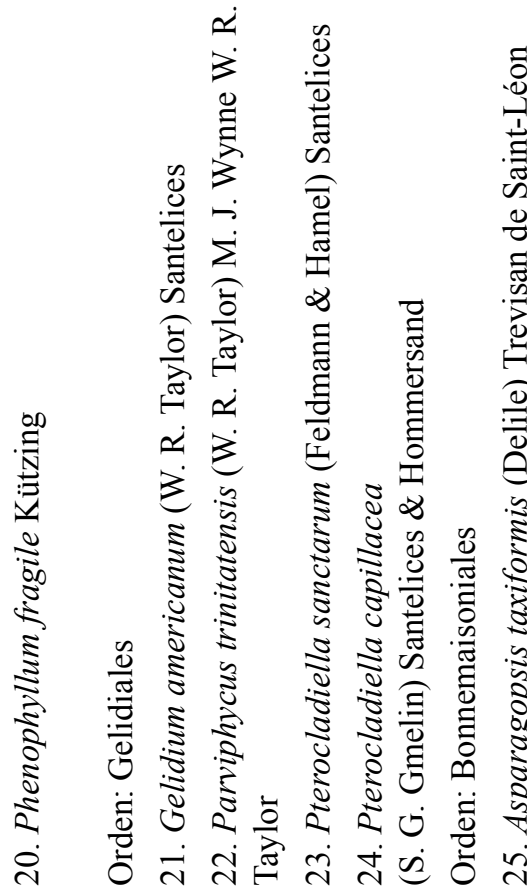



$\sum^{n}$

E

芒

苞是

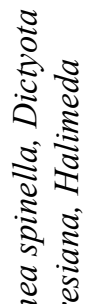

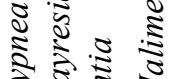



I

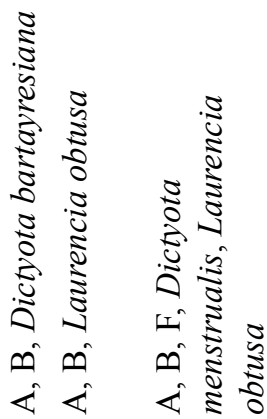

궁 ণ

$\stackrel{\infty}{3}$

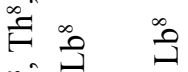

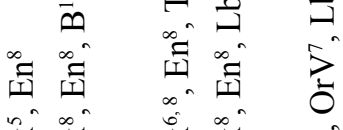
苍爻



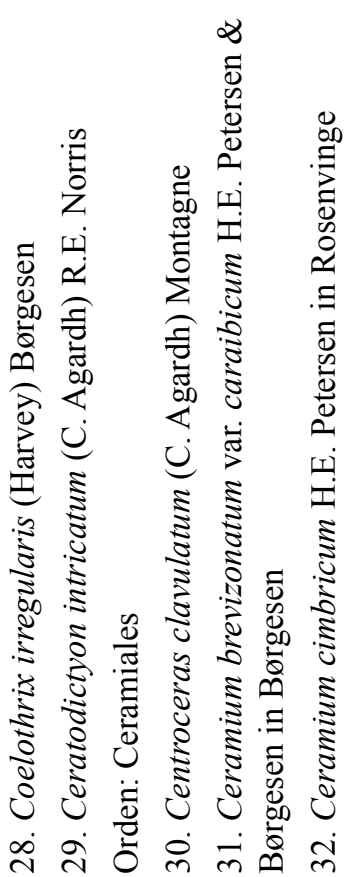





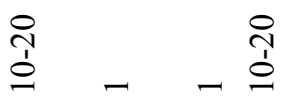

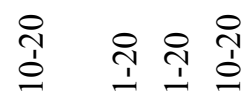

ิㅗㅇ 옹

ิㅗㅇ

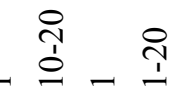

3

$\sum^{\infty}$

$i^{\infty}$

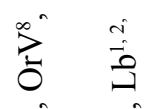

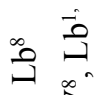

है

$\bar{n}$

울

Е $\stackrel{\infty}{\equiv} \cong$

$\stackrel{\infty}{\equiv}$

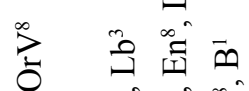



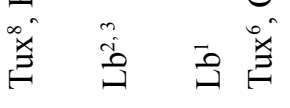

ज्ञ

䓂爻笔



$\infty \infty^{\infty} \infty$

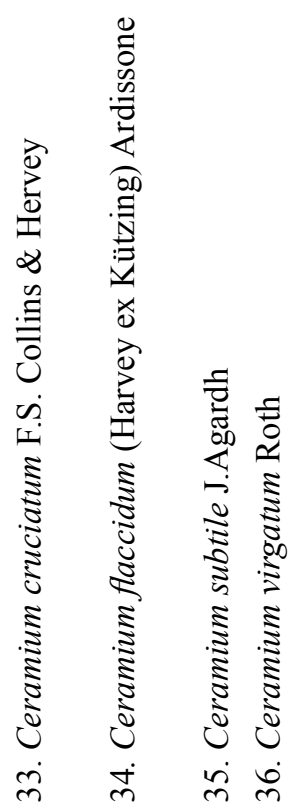

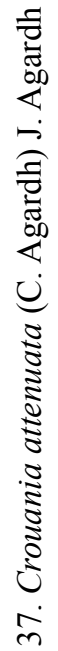

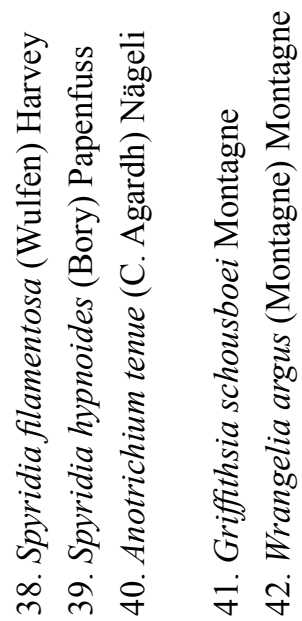

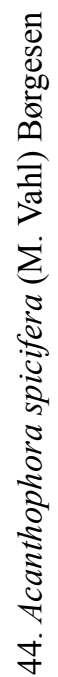

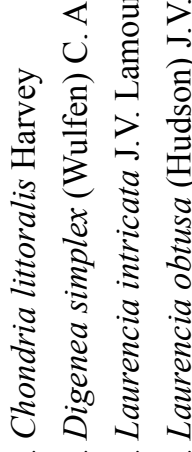

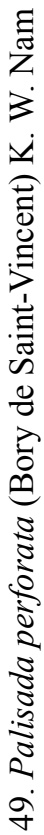




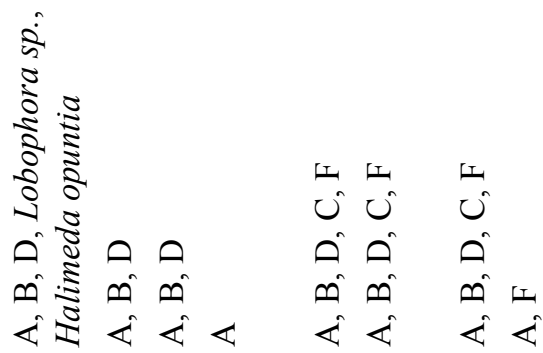

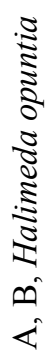

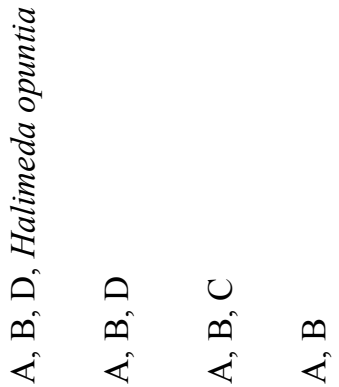

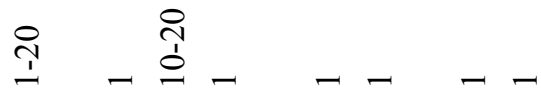

$\begin{array}{lllll}\text { กิ } & \text { กิ } \\ 1 & 1 & - & -\end{array}$

$\sum_{i=1}^{\infty}$

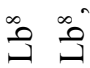

$\stackrel{\infty}{1}$

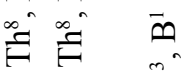

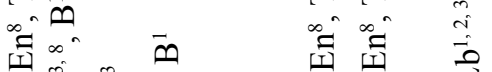

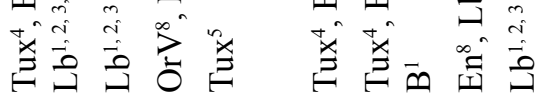

$\sum_{i=1}^{n}$

龺

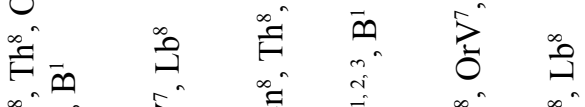

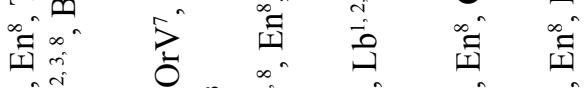
至苟
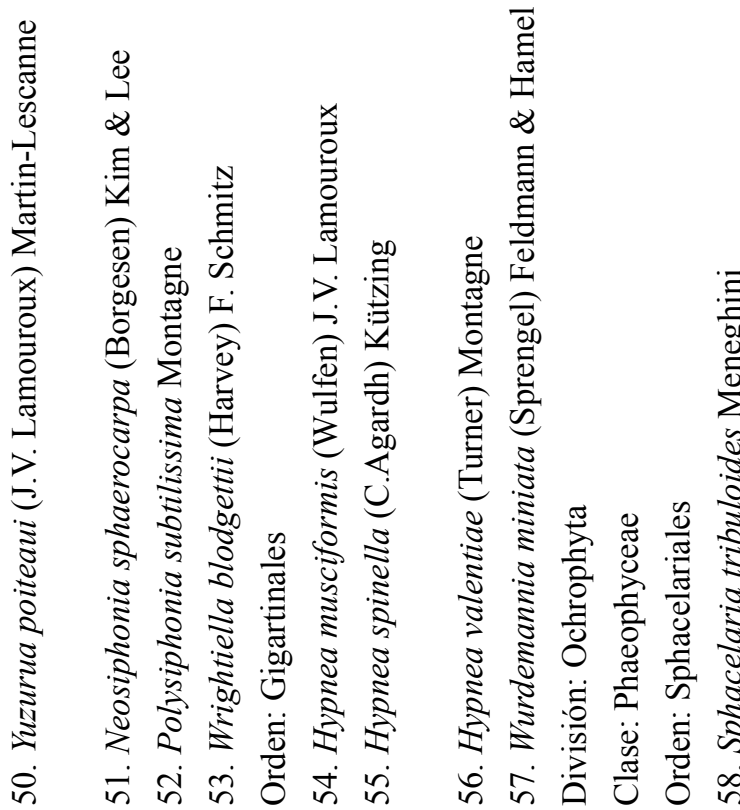




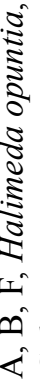

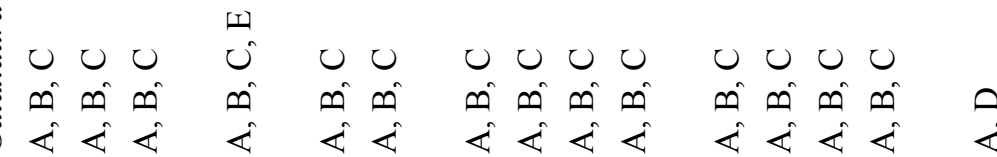

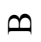

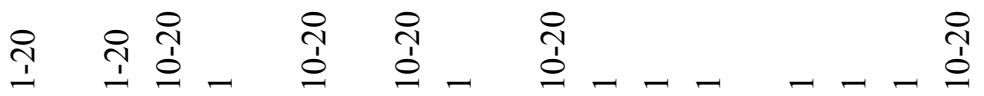



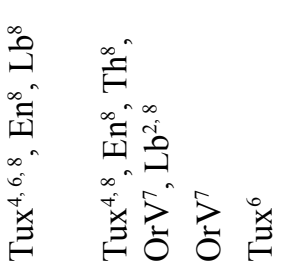

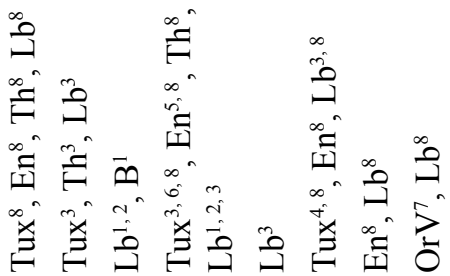

${ }_{0}^{\infty}=$

อ
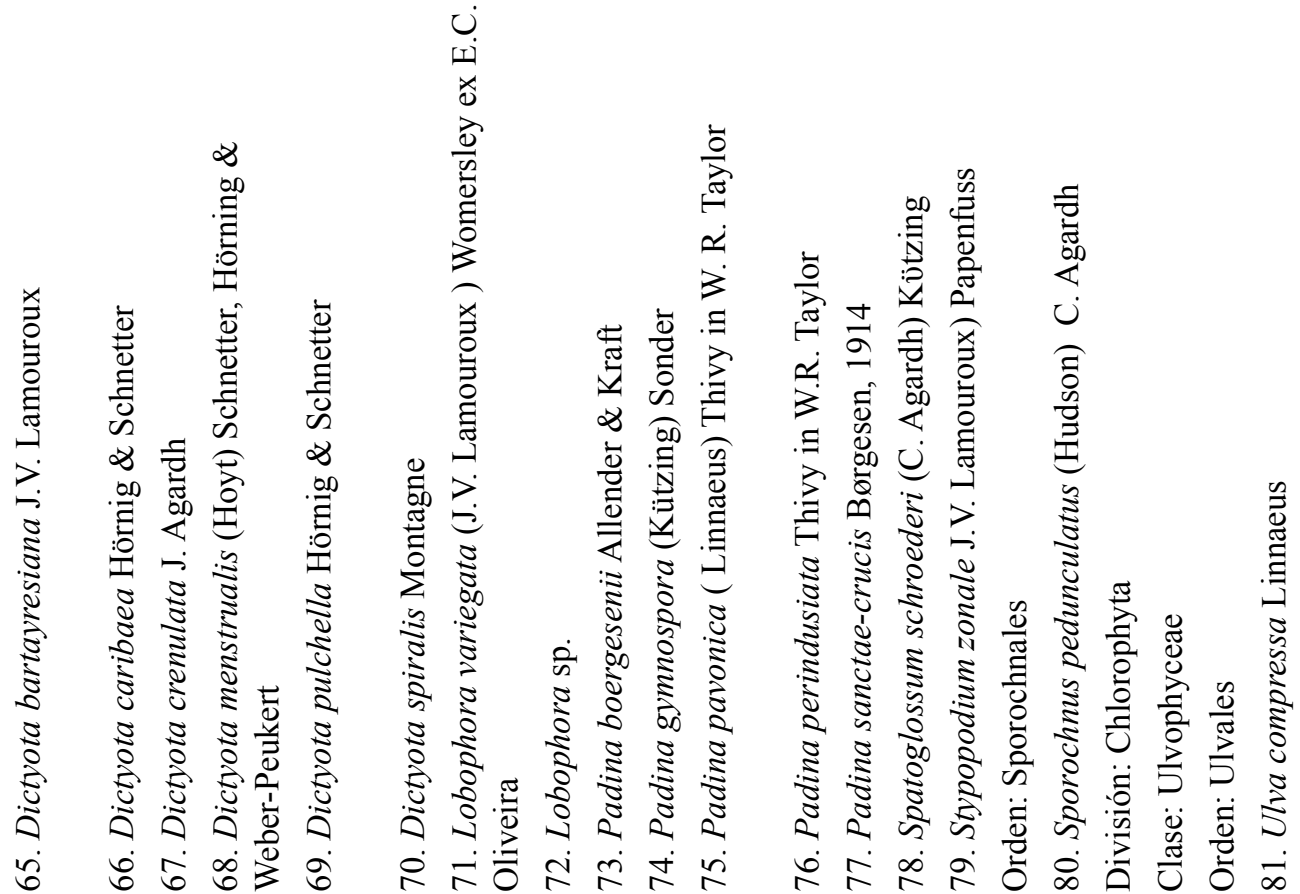


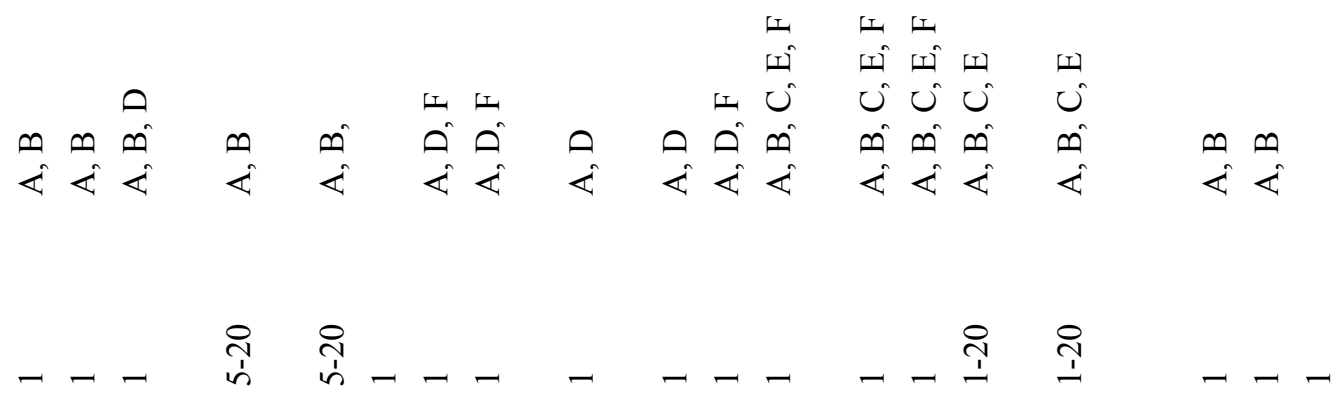

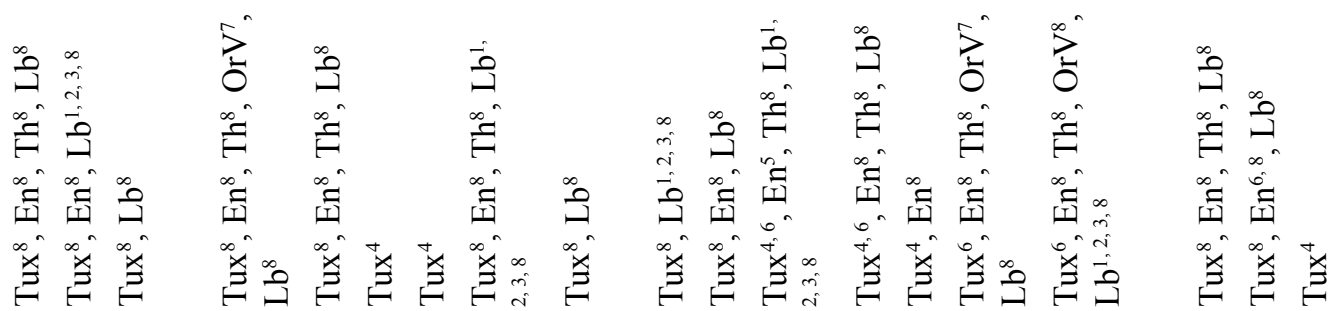






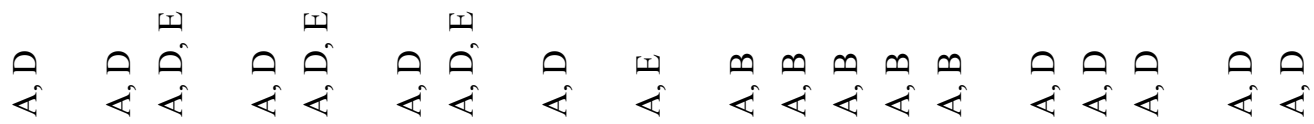



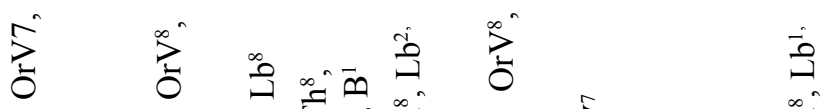

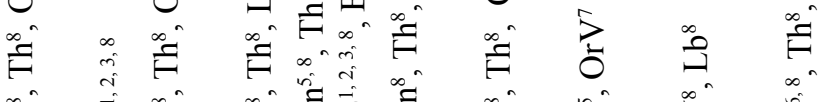

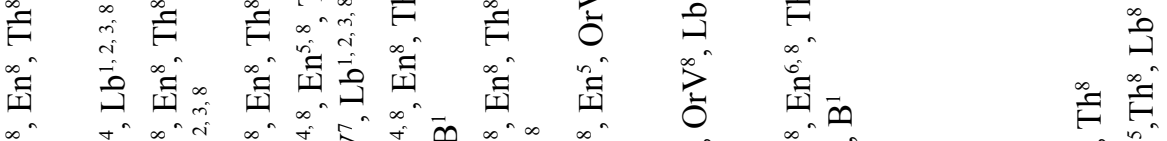
秜 馬

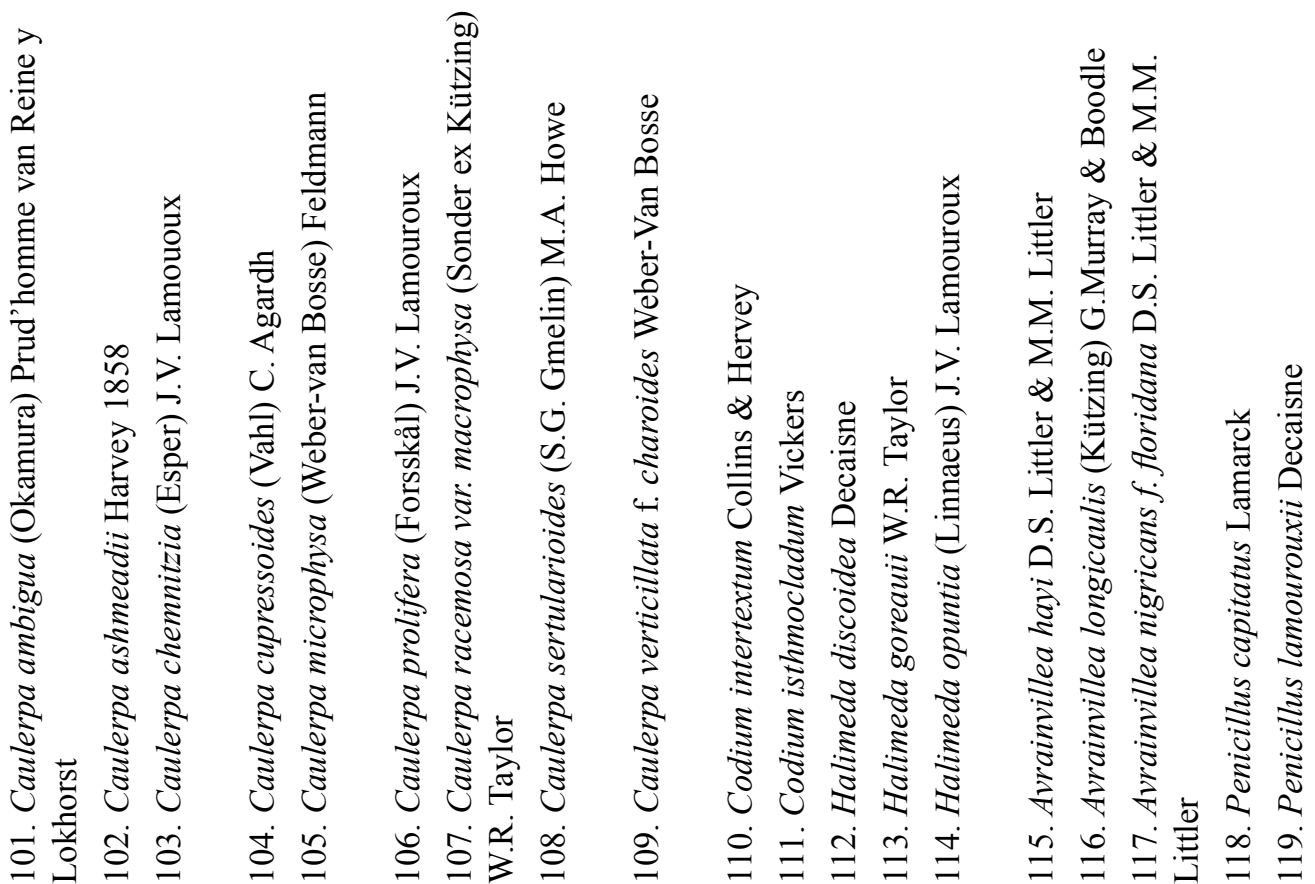




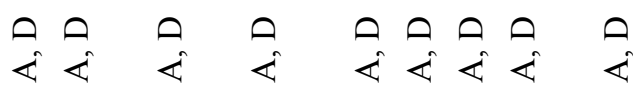

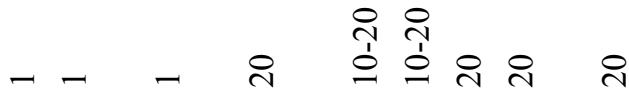



ㄱ-
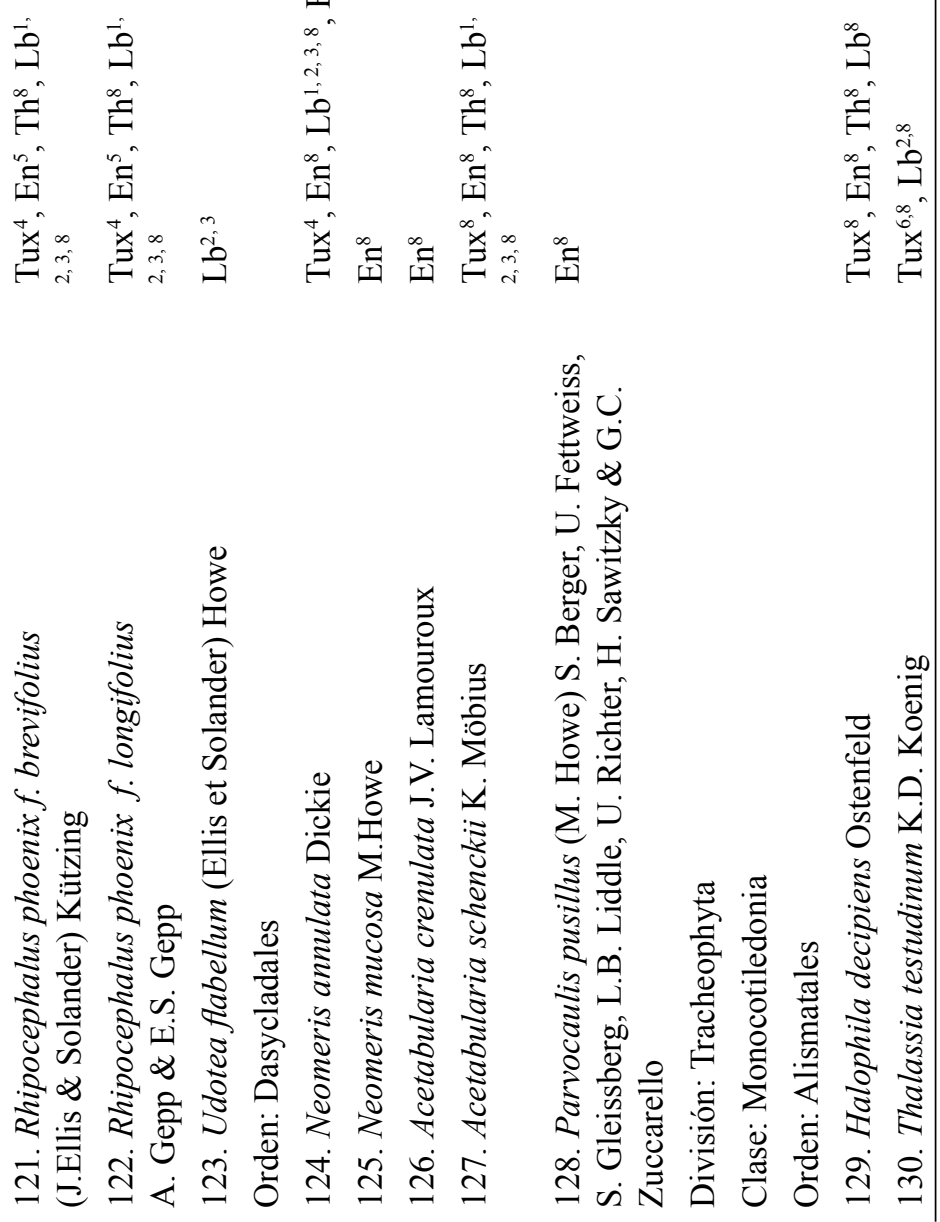
A escala de arrecife, el número de especies se incrementó de manera importante, en especial para el arrecife Enmedio, se reportaban en la literatura 11 especies y en este estudio se encontraron 74 registros nuevos lo que aumenta la riqueza florística a 80 especies, así mismo, para el arrecife Lobos de 55 especies (reportadas anteriormente) aumentó a 91 taxa. Mientras, las 53 especies que se observaron en el arrecife Tanhuijo constituyen los primeros registros, en cambio, las 27 especies que presenta el arrecife Blanquilla son registros que fueron obtenidos de la literatura (Cuadro 1 y 2).

Al comparar la riqueza de especies en el nivel de arrecife, los de tipo emergente con mayor riqueza (>80 especies) fueron Lobos, Tuxpan y Enmedio, por el contrario, el Blanquilla tiene menos registros de especies, al igual que el arrecife sumergido Oro Verde con 43 especies de algas (Cuadro 1). En orden de importancia, Chlorophyta seguido de Rhodophyta fueron las más representativas en los arrecifes emergentes, por el contrario, para el arrecife sumergido Oro Verde la riqueza de Chlorophyta decrece y prevalece Rhodophyta (Cuadro 1 y 2).

Por otra parte, de las 55 especies de Rhodophyta registradas en este estudio, al menos 21 especies se observaron en 4 arrecifes del SALT, la mayoría de las especies se encontraron en rocas coralinas y guijarros (Cuadro $2)$. En la laguna arrecifal (1 $\mathrm{m}$ de profundidad) se encontraron 24 especies, entre las comunes Amphiroa fragilissima y Acanthophora spicifera, así como también especies del género Ceramium, Laurencia e Hypnea (Cuadro 2). Mientras, otras 16 se observaron entre $1-20 \mathrm{~m}$ de profundidad (Cuadro 2), por ejemplo, Dichotomaria marginata (Fig. 2A) y Galaxaura rugosa (Fig. 2B). Entre $\operatorname{los} 5$ y $20 \mathrm{~m}$ de profundidad, se contemplaron principalmente 2 especies: Parviphycus trinitatensis y Predaea feldmannii (Fig. 2E). En cambio, en las zonas más profundas $(10-20 \mathrm{~m})$ se encontraron 14 especies (Cuadro 2), siendo comunes Tricleocarpa cylindrica (Fig. 2C), Asparagopsis taxiformis (Fig. 2D) y Dictyurus occidentalis (Fig. 2F). Cabe resaltar que 19 especies de algas rojas se encontraron como epifitas de otras macroalgas rojas, pardas y verdes (e. g. Galaxaura, Padina, Laurencia, Halimeda) (Cuadro 2).

En relación con las Ochrophyta (algas pardas), solamente 6 especies se registraron en al menos 4 arrecifes del SALT. Principalmente estas algas pardas se establecen en rocas coralinas y guijarros, distribuyéndose más de la mitad de las especies en la laguna arrecifal (Cuadro 2), donde las más comunes de observar son Colpomenia sinuosa, Dictyota menstrualis y Padina gymnospora. Otras especies como Sphacelaria tribuloides y Dictyota bartayresiana (Fig. 2G), se 





Fig. 2. Especies de algas y fanerógamas del Sistema Arrecifal Lobos-Tuxpan, México. A. Dichotomaria marginata. B. Galaxaura rugosa. C. G. subverticillata. D. Asparagopsis taxiformis. E. Predaea feldmannii. F. Dictyurus occidentalis. G. Dictyota bartayresiana. H. D. pulchella. I. Stypopodium zonale. J. Anadyomene saldanhae. K. A. stellata. L. Chaetomorpha gracilis. M. Dictyosphaeria cavernosa. N. D. ocellata. Ñ. Valonia macrophysa. O. V. ventricosa. P. Bryopsis plumosa. Q. Caulerpa chemnitzia. R. C. cupresoides. S. C. microphysa. T. C. prolifera. U. C. racemosa. V. C. sertularioides. W. C. verticillata. X. Codium intertextum. Y. Halimeda discoidea. Z. H. opuntia. AA. Avrainvillea hayi. AB. Rhipocephalus phoenix f. brevifolius. AC. Neomeris annulata. AD. N. mucosa. AE. Acetabularia schenckii. AF. Parvocaulis pusillus. AG. Halophila decipiens. AH. Thalassia testudinum

Fig. 2. Algae and phanerogam species of the Lobos-Tuxpan Reef System, Mexico. A. Dichotomaria marginata. B. Galaxaura rugosa. C. G. subverticillata. D. Asparagopsis taxiformis. E. Predaea feldmannii. F. Dictyurus occidentalis. G. Dictyota bartayresiana. H. D. pulchella. I. Stypopodium zonale. J. Anadyomene saldanhae. K. A. stellata. L. Chaetomorpha gracilis. M. Dictyosphaeria cavernosa. N. D. ocellata. N. Valonia macrophysa. O. V. ventricosa. P. Bryopsis plumosa. Q. Caulerpa chemnitzia. R. C. cupresoides. S. C. microphysa. T. C. prolifera. U. C. racemosa. V. C. sertularioides. W. C. verticillata. X. Codium intertextum. Y. Halimeda discoidea. Z. H. opuntia. AA. Avrainvillea hayi. AB. Rhipocephalus phoenix f. brevifolius. AC. Neomeris annulata. AD. N. mucosa. AE. Acetabularia schenckii. AF. Parvocaulis pusillus. AG. Halophila decipiens. AH. Thalassia testudinum observaron en las áreas someras y profundas de los arrecifes, en cambio, Dictyota pulchella (Fig. 2H), Stypopodium zonale (Fig. 2I) y Sphorocnus pedunculatus tienen distribución en las zonas profundas de los arrecifes (Cuadro 2).

Respecto a

las Chlorophyta, 20 especies fueron observadas en al menos cuatro arrecifes del SALT, donde el $56 \%$ del total de especies son propias de la laguna arrecifal (Cuadro 2), siendo las más frecuentes de avistar: Chaetomorpha gracilis (Fig. 2L), Dictyosphaeria cavernosa (Fig. 2M), Dictyosphaeria ocellata (Fig. 2N), Bryopsis plumosa (Fig. 2P), Caulerpa cupresoides (Fig. 2R), Caulerpa racemosa (Fig. 2U), Caulerpa sertularioides (Fig. 2V), Halimeda opuntia (Fig. 2Z) y Rhipocephalus phoenix (Fig. 2AB). En cambio, algunas especies comunes como Valonia macrophysa (Fig. 2N) y Valonia ventricosa (Fig. 2O) se registraron en la planicie como en las zonas profundas de $20 \mathrm{~m}$ (Cuadro 2). Así también, varias especies de algas verdes se observaron únicamente en las pendientes profundas (Cuadro 2), entre las que se pueden mencionar son: Anadyomene 
saldanhae (Fig. 2J) Anadyomene stellata (Fig. 2K), Caulerpa chemnitzia (Fig. 2Q), Caulerpa microphysa (Fig. 2S), Caulerpa verticillata (Fig. $2 \mathrm{~W}$ ) y Codium intertextum (Fig. 2X), las cuales se establecen en roca coralina. Mientras, Caulerpa prolifera (Fig. 2T), Halimeda discoidea (Fig. 2Y), Avrainvillea hayi (Fig. 2AA), Neomeris mucosa (Fig. 2AC), Neomeris annulata (Fig. 2AD), Acetabularia schenckii (Fig. 2AE) y Parvocaulis pusillus (Fig. 2AF) son típicos en zonas de arena del talud arrecifal (Cuadro 2).

En cuanto a las fanerógamas marinas, se registra por primera vez para la zona Halophila decipiens (Fig. 2AG), esta especie se distribuye en el talud arrecifal de sotavento de los arrecifes emergentes. También se registró la presencia de Thalassia testudinum (Fig. 2AH) principalmente en la laguna arrecifal de Tuxpan y Lobos (Cuadro 2).

\section{Nuevo registro para el suroeste del golfo de México}

Clase Phaeophyceae Orden Sporochnales

Familia Sporocnaceae Género Sporochnus C.Agardh

\section{Sporochnus pedunculatus}

(Hudson) C.Agardh

Basiónimo: Fucus pedunculatus

Hudson

Fig. 3A-G
Material examinado. CBUV00020, arrecife Enmedio (21.082253 ${ }^{\circ}$ $\mathrm{N},-97.258003^{\circ} \mathrm{W}$ ), recolectado a $12 \mathrm{~m}$ de profundidad, col. V. De la Cruz-Francisco, 18 junio, 2014.

Descripción. Frondas de color amarillento observadas en vivo (Fig. 3A), y amarillo ocre conservadas en formol (Fig. 3B). Talo principal de textura lisa, cilíndrico de $1 \mathrm{~mm}$ de diámetro y 30-40 cm de altura, sujeto al sustrato por un disco basal. Células medulares de 36.4-55.8 $\mu \mathrm{m}$ de diámetro y de 60.5-120.8 $\mu \mathrm{m}$ de largo (Fig. 3C-D). Células periféricas de 8.7-11.5 $\mu \mathrm{m}$ de diámetro (Fig. 3D). Ramas secundarias de $0.7-1 \mathrm{~mm}$ de diámetro y de 4-6 cm de largo (Fig. 3E-1), las cuales se distribuyen de manera helicoidal a lo largo de los talos. En fase reproductiva presentan un pedicelo y un mechón terminal de filamentos color pardo (Fig. 3E-3). Cada rama posee de 16-28 receptáculos, estas estructuras son gruesas y ovoides de 0.3-0.7 mm diámetro y de 1.5-2.3 mm de largo (Fig. 3E-2). Los filamentos se extienden de 3-7 mm, lo componen células de $17-20 \mu \mathrm{m}$ de largo y $4.5-5 \mu \mathrm{m}$ de ancho (Fig. 3E-G).

Hábitat. Sustrato arenoso y roca coralina, a $12 \mathrm{~m}$ de profundidad.

Distribución geográfica. Especie de amplia distribución, Europa, Asia, África (Fredericq et al. 2009). En el Atlántico Occidental, presente en Cuba (Alfonso et al. 2014; Suárez et al. 2015) y en el Caribe (Littler \& 

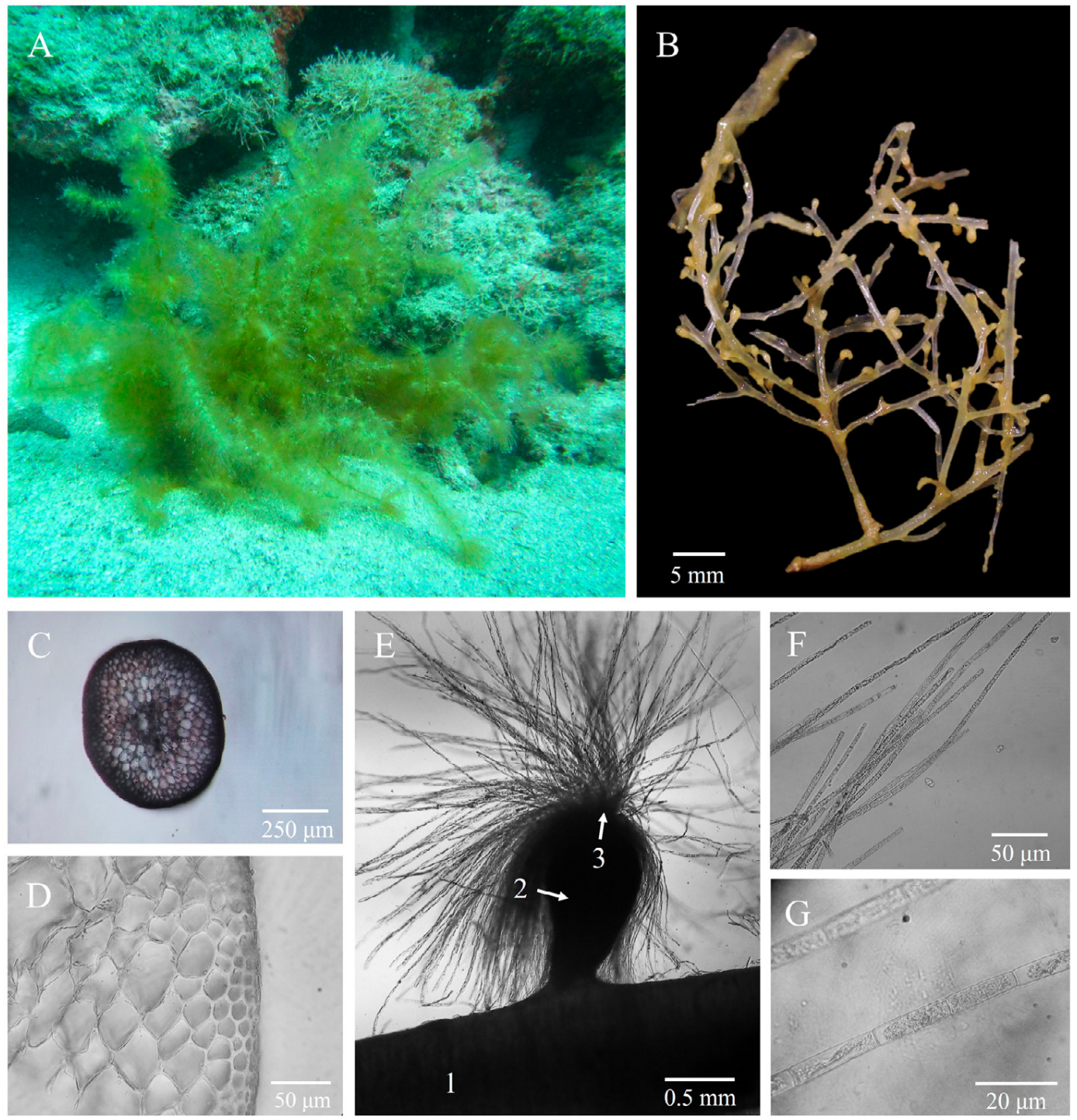

Fig. 3. A. Presencia Sporochnus pedunculatus en el arrecife Enmedio. B. Ramas secundarias con receptáculos. C. Corte transversal del talo. D. Células medulares y periféricas del talo. E. 1. Superficie de rama secundaria. 2. Receptáculo. 3. Mechón filamentoso. F-G. Vista aumentada de células que componen el mechón

Fig. 3 A. Presence of Sporochnus pedunculatus in the Enmedio reef. B. Secondary branches with receptacles. C. Cross section of the talus. D. Medullary and peripheral cells of the talus. E. 1. Secondary branch area. 2. Receptacle. 3. Filamentous tuft. F-G. Magnified view of cells that make up the tuft 
Littler, 2000; Fredericq et al. 2009). México: Yucatán (Huerta-Musquiz et al. 1987). Se cita por primera vez para el suroeste del golfo de México.

Comentarios. Las características morfológicas del espécimen estudiado coinciden con las descripciones de Brostoff, (1984), Boraso \& Negri (1997), Littler \& Littler (2000) y Alfonso et al. (2014). En el golfo de México, además de $S$. pedunculatus, se ha reportado otra especie más, Sporochnus bolleanus Montagne con distribución para el sureste del golfo (Fredericq et al. 2009), sin embargo, esta especie presenta mayor longitud en el pedicelo $(6-8 \mathrm{~mm})$, receptáculo (1-10 mm) y en los filamentos del penacho (10-15 mm) (Brostoff, 1984).

\section{DISCUSIÓN}

El presente trabajo aporta una lista actualizada de la flora bentónica del SALT, el cual está compuesta por 130 especies. Esta riqueza florística representa un buen avance para el área natural protegida, dado que previamente se reportaban en la literatura 102 especies que correspondían en su mayoría al arrecife Lobos y Tuxpan. Sin embargo, dentro del corredor arrecifal, en el sistema arrecifal SAV se tienen registradas 280 especies hasta el 2019 (Galicia-García \& Morales-García, 2007; Robinson et al. 2012; Galicia-García et al. 2013; Godínez-Ortega et al. 2015; 2019), por lo que, se considera que el inventario florístico del SALT puede estar aún incompleto, razón por la cual se requiere mayor número de estudios encaminados a inventariar y explorar arrecifes poco conocidos de esta zona arrecifal.

En las lagunas arrecifales del SALT se observó mayor riqueza de algas, lo cual se puede atribuir a que es una zona protegida del oleaje, que a diferencia de la cresta arrecifal donde las especies están expuestas al estrés ambiental por el embate de las olas (Lehman, 2010), también se puede atribuir a la existencia de una amplia variedad de sustratos como rocas, guijarros, arena y pastos marinos, los cuales son utilizados por las propias algas para fijarse (González-Gándara et al. 2007). En cambio, en las pendientes profundas se registró menor número de especies de algas, lo cual puede estar asociado al tipo de fondo que principalmente es sustrato duro y con corales masivos donde esencialmente se establecen algas rojas filamentosas (González-González et al. 2016), las cuales forman tapetes que cubren las rocas coralinas y las superficies muertas de los corales (Lehman, 2010; Luna \& De la Cruz-Francisco, 2017).

Las algas rojas encabezan el grupo con mayor riqueza de especies para el SALT, lo cual es normal, dado que es el grupo más diverso en especies en el golfo de México (Ortega et al. 2002; Fredericq et al. 2009). No obstante, dentro del corredor arrecifal, se 
han reportado mayor número de especies de algas rojas en el SAV, siendo las familias más representativas: Corallinacaea, Gelidiaceae, Gracilariaceae, Ceramiaceae, Rhodomelaceae, (Galicia-García \& Morales-García, 2007), por lo tanto, se recomienda un mayor esfuerzo de trabajo de tipo taxonómico, con la finalidad de contribuir con más registros de este grupo de algas para la zona de estudio, además es un grupo difícil de identificar y que requiere de estudios detallados (Lehman, 2010).

Por otra parte, varias especies de rodófitas (G. rugosa, Amphiroa y Laurencia), ocrofitas (Padina gymnospora y Dictyota menstrualis) y clorófitas $(C$. sertulariodes, $C$. racemosa y $D$. cavernosa, H. opuntia y R. phoenix) observadas en zonas protegidas de la laguna arrecifal denotan una típica zonación que ha sido descrita previamente por Lehman (2010) para el arrecife Isla de Enmedio. No obstante, la distribución de las macroalgas depende del tipo de sustrato (Garduño-Solórzano et al. 2005) y las rocas coralinas parecen ser uno de los sustratos más importantes para las macroalgas, dado que se registró la mayor cantidad de especies en el presente estudio, estos sustratos son duros, consolidados y por su textura rugosa favorecen el desarrollo de algas (Garduño-Solórzano et al. 2005; González-Gándara et al. 2007; Martínez-Daranas et al. 2016).
Particularmente, Valonia spp y Dictyosphaeria spp fueron encontradas en los intersticios de las rocas coralinas y entre colonias de coral, habitualmente es una estrategia para protegerse del oleaje (Garduño-Solórzano et al. 2005). Así también, Dictyota menstrualis, Dictyota bartayresiana, Caulerpa racemosa, Caulerpa cupressoides y Halimeda opuntia fueron comunes en zonas dominadas por corales escleractinios (Porites spp y Pseudodiploria spp), estos resultados coinciden con lo reportado por Mateo-Cid et al. (1996) para el arrecife Isla Verde y por González-Gándara et al. (2007) para el arrecife Tuxpan.

La arena constituye un buen sustrato para las Chlorophyta, principalmente las que presentan mecanismos de adaptación para anclarse al sustrato e. g. Caulerpa, Halimeda y Rhipocephalus (Garduño-Solorzano et al. 2005). En las lagunas arrecifales del SALT, fue común observar varias especies de algas verdes psamófilas como $C$. racemosa, C. sertularioides, H. opuntia, Avrainvillea nigricans y $R$. phoenix, estas especies también son típicas en otras regiones del golfo de México (Mateo-Cid et al. 1996; 2013; Garduño-Solorzano et al. 2005). Sin embargo, en las zonas arenosas profundas del SALT, la composición de especies psamólifas está representado por otras: Halimeda discoidea, Avrainvillea hayi, Neomeris annulata, Acetabularia schenckii y Parvocaulis 
pusillus, las cuales pueden distribuirse en profundidades $>30 \mathrm{~m}$ (Littler \& Littler, 2000).

Cabe mencionar que especies como Caulerpa chemnitzia, Caulerpa microphysa, Caulerpa verticillata, Codium intertextum, $A$. saldanhae, $H$. discoidea, A. taxiformis y $S$. zonale, fueron observadas particularmente en las zonas profundas de los arrecifes del SALT, esta distribución batimétrica parece ser normal dado que en áreas del Caribe y la Florida este ensamblaje de macroalgas se han encontrado a más de $60 \mathrm{~m}$ de profundidad (Littler \& Littler, 2000; Leichter et al. 2008).

Respecto a las fanerógamas marinas, de las nueve especies que se reportan para México (Atlántico y Pacifico) (Lot-Helgueras, 1971; Cervantes-Maldonado \& Quintero, 2016), se encontraron en este estudio: $\mathrm{Ha}$ lophila decipiens y Thalassia testudinum. La única que se observó en la mayoría de los arrecifes del SALT fue $H$. decipiens, principalmente en zonas arenosas de sotavento, mientras, $T$. testudinum se encontró, en especial, en el arrecife Lobos, donde ocupa extensas áreas en la laguna arrecifal (Chávez et al. 1970), también se observó en el Tuxpan, pero presenta un reducido parche al oeste de la planicie arrecifal (González-Gándara et al. 2007; Aguilera-Pérez \& De la Cruz-Francisco, 2017). Ambas especies de pastos son las únicas de la flora marina del SALT que presentan un estatus de riesgo dado que están catalogadas en la NOM-059-SEMARNAT-2010 con la categoría de protección especial (DOF, 2019), por lo que es necesario redoblar esfuerzos para estudiar la estructura poblacional de cada una de estas fanerógamas marinas.

Si bien, este trabajo documenta la riqueza florística de al menos cinco de los diez arrecifes que se distribuyen en el norte de Veracruz, aún es necesario dirigir más estudios en arrecifes que no han sido explorados como los arrecifes que recientemente han sido cartografiados: arrecife Corazones y Pantepec Sur, además de los arrecifes Blake y Piedras Altas, estos últimos localizados frente a las costas de los municipios de Cazones y Tecolutla (Ortiz-Lozano et al. 2019).

Por último, el presente trabajo sienta las bases para continuar con más estudios relacionados a la flora marina bentónica en los arrecifes del SALT, a fin de obtener una lista completa de las especies, de esta manera se podrá realizar comparaciones de la riqueza, distribución y conectividad con los demás arrecifes coralinos del golfo de México. Así también, la presente información puede ser de utilidad en materia de manejo y protección en especial para salvaguardar las especies en riesgo como los pastos marinos. Adicionalmente, la actual lista de algas y fanerógamas del SALT evidencia resultados positivos, con un incremento considerable de la riqueza florística para los arrecifes 
Lobos, Enmedio y Tuxpan, no obstante, también expone huecos de información como sucede con los arrecifes Blanquilla y Medio.

\section{AGRADECIMIENTOS}

Agradecemos a Itzel Morales Quijano, Irving Abad Aguilar, Ivonne Luna Ortega y Víctor Alfonso Reyna Matezans por su apoyo en las labores de campo. A los revisores anónimos por sus comentarios y sugerencias.

\section{REFERENCIAS}

Aguilar-Rosas, R., Aguilar-Rosas, L. E., Ávila S. G. E., González Y. O. \& Becerril B. F. (2010). Macroalgas submareales de la bahía de todos Santos, Baja California, México. Rev. Mex. Biodiv., 81(3), 601-6018. http://dx.doi.org/10.22201/ ib.20078706e.2010.003.632

Aguilera-Pérez, A. \& De la Cruz-Francisco, V. (2017). Composición taxonómica de las algas césped y su macrofauna asociada, arrecife Tuxpan, Sistema Arrecifal Lobos-Tuxpan. Bol. Inst. Oceanogr. Venezuela, 52(2), 66-80.

Alfonso S., Y., Martínez-Daranas, B., Suárez A. A. M. \& Rey-Villiers, N. (2014). Nuevos registros para la flora marina bentónica cubana. Rev. Invest. Mar., 34(1), 18-23.

Alves, A. M., Gestinari, L. M. \& Moura, C. W. D. N. (2010). La familia Valoniaceae (Chlorophyta) en el estado de Bahía, Brasil: aspectos morfológicos y de distribución. Hidrobiológica, 20(2), 171-184.

Arellano-Méndez, L. U., Bello-Pineda, J., Aké-Castillo, J. A., Pérez-España, H.
\& Martínez-Cárdenas, L. (2016). Distribución espacial y estructura morfométrica de las praderas de Thalassia testudinum (Hydrocharitaceae) en dos arrecifes del Parque Nacional Sistema Arrecifal Veracruzano, México. Rev. Biol. Trop., 64(2), 427-448. https://doi. org/10.15517/rbt.v64i2.19810

Bandeira-Pedrosa, M. E., Pereira, S. M. B. \& Oliveira, E. C. (2004). Taxonomy and distribution of the Green algal genus Halimeda (Bryopsidales, Chlorophyta) in Brazil. Braz. J. Bot., 27(2), 363-377. https://doi.org/10.1590/ s0100-84042004000200015

Boraso, A. L. \& Negri, R. (1997). Presencia de Sporochnus pedunculatus (Sporochnales, Phaeophycophyta) en la costa Argentina. Physis (Buenos Aires), 54(126-127), 23-24.

Brostoff, W. N. (1984). Sporocnhus dotyi sp. nov. (Sporochnales, Phaeophyta), a brown alga from Hawaii. Pac. Sci., 38(2), 177-181.

Cervantes-Maldonado, A. \& Quintero, E. (2016). La importancia de conservar las praderas de pastos marinos. CONABIO Biodiversitas, 128,12-16.

Chávez, E. A., Hidalgo, E. \& Sevilla, M. L. (1970). Datos acerca de las comunidades bentónicas del arrecife de Lobos, Veracruz. Rev. Soc. Mex. Hist. Nat., 31, 211-80.

De la Cruz-Francisco, V., González-González, M. \& Flores-Galicia, L. (2016). Distribución de los hábitats bentónicos de la laguna del arrecife Enmedio, Sistema Arrecifal Lobos-Tuxpan, México. Rev. Invest. Mar., 36(1), 63-78.

DOF. (2009). Decreto por el que se declara área natural protegida, con el carácter de Área de Protección de Flora y Fauna, la región conocida como Sistema Arrecifal Lobos-Tuxpan, localizada frente a las costas de los municipios 
de Tamiahua y Tuxpan, en el Estado de Veracruz. Diario Oficial de la Federación. https://simec.conanp.gob.mx/ pdf_decretos/132_decreto.pdf.

DOF. (2019). Modificación del anexo normativo III, lista de especies en riesgo de la Norma Oficial Mexicana NOM-059-SEMARNAT-2010, protección ambiental-especies nativas de México de flora y fauna silvestres-categorías de riesgo y especificaciones para su inclusión, exclusión o cambio lista de especies en riesgo. Diario Oficial de la Federación, https://www. dof.gob.mx/nota_detalle.php?codigo $=5578808 \&$ fecha $=14 / 11 / 2019$.

Flores-Leiva, L., Gavio, B., Díaz-Ruiz, M., Camacho, O. \& Díaz-Pulido, G. (2010). Recolección y preservación de macroalgas marinas: una guía para estudios ficológicos. Rev. Intropica, 5, 97-103.

Fong, P. \& Paul, V. J. (2011). Coral reef algae. In: Z. Dubinsky \& N. Stamber (Eds), Coral reefs: an ecosystem in transition (pp. 241-272). New York: Springer. https://doi. org/10.1007/978-94-007-0114-4_17

Fredericq, S., Cho, T. O., Earle, S. A., Gurgel, C. F., Krayesky, D. M., Mateo-Cid, L. E., Mendoza-González, A. C., Norris, J. N. \& Suárez, A. M. (2009). Seaweeds of the Gulf of Mexico. In D. L. Felder \& D.K. Camp (Eds.), Gulf of Mexico-Origins, Waters, and Biota. Biodiversity (pp. 187-259). E.E. U.U. Texas A \& M Press, College Station.

Galicia-García, C. \& Morales-García, A. (2007). Investigaciones sobre macroalgas realizadas en el sistema arrecifal veracruzano. En A. Granados-Barba, L. G. Abarca-Arenas \& J. M. Vargas-Hernández (Eds.), Investigaciones cientificas en el Sistema Arrecifal Veracruzano (pp. 141-160). México: Universidad Autónoma de Campeche.
Galicia-García, C., Robinson, N. M. \& Okolodkov, Y. (2013). New records of red algae (Rhodophyta) for Cabezo reef, Natinal Park Sistema Arrecifal Veracruzano, Gulf of Mexico. Acta Bot. Mex., 102, 39-76.

Garduño-Solórzano, G., Godínez-Ortega, J. L. \& Ortega, M. M. (2005). Distribución geográfica y afinidad por el sustrato de las algas verdes (Chlorophyceae) bénticas de las costas mexicanas del golfo de México y mar Caribe. $B$. Soc. Bot. Mex., 76, 61-78. https://doi. org/10.17129/botsci.1705

Godínez-Ortega, J. L., Ramírez-García, P. \& Granados-Barba, A. (2015). Estructura comunitaria del macrofitobentos en los arrecifes De Enmedio y Chopas. En A. Granados-Barba, L. Ortíz-Lozano, D. Salas-Monreal \& C. González-Gándara (Eds.), Aportes al conocimiento del Sistema Arrecifal Veracruzano: hacia el Corredor Arrecifal del Suroeste del Golfo de México (pp. 35-46). México: Universidad Autónoma de Campeche.

Godínez-Ortega, J. L., Ramírez-García, P., Granados-Barba, A. \& Wynne, M. (2019). New records of subtidal benthic marine algae from the state of Veracruz, southern Gulf of Mexico. Rev. Mex. Biodivers., 90: e902719. http://dx.doi.org/10.22201/ ib.20078706e.2019.90.2719

González-Gándara, C., M. Cruz-Arellano, C. Domínguez-Barradas, A. Serrano-Solís \& Basáñez-Muñoz, A. J. (2007). Macroalgas asociadas a cuatro hábitats del Arrecife de Tuxpan, Veracruz, México. UDO Agrícola, 7(1), 252-257.

González-González, M., De La Cruz-Francisco, V., Morales-Quijano, I. \& Orduña-Medrano, R. E. (2016). Diversidad y cobertura de la comunidad bentónica del arrecife Enmedio, Sistema Arrecifal Lobos-Tuxpan, México. Rev. 
Mar. Cost., 8(2), 47-65. https://doi. org/10.15359/revmar.8-2.3

Guiry, M. D. \& Guiry, G. M. (2019). AlgaeBase. World-wide electronic publication, National University of Ireland, Galway. http://www.algaebase.org.

Huerta-Múzquiz, L. \& Barrientos, A. C. (1965). Algas marinas de la Barra de Tuxpan y de los arrecifes Blanquilla y Lobos. An. Esc. Nac. Cienc. Biol., 13(1-4), 5-21.

Huerta-Múzquiz, L., Mendoza González, A. C. \& Mateo Cid, L. E. (1987). Avance sobre un estudio de las algas marinas de la Península de Yucatán. Phytologia, 62, 23-53.

Ibarra-Morales, N. \& Abarca Arenas, L. G. (2007). Distribución, abundancia y biomasa de Thalassia testudinum en la laguna del arrecife Sacrificios, Veracruz. En A. Granados Barba, L. G. Abarca Arenas \& J. M. Vargas Hernández (Eds.), Investigaciones Cientificas en el Sistema Arrecifal Veracruzano (pp. 161-172), México: Universidad Autónoma de Campeche.

Lehman, R. L. (2010). Algas de los arrecifes. En J.W. Tunnell Jr., E.A. Chávez \& K. Withers (Eds), Arrecifes Coralinos del Sur del Golfo de México (pp. 129-140). México: Instituto Politécnico Nacional.

Leichter, J. J., Dale S., M. \& Genovese, S. J. (2008). Deep water macroalgal communities adjacent to the Florida Keys reef tract. Mar. Ecol. Prog. Ser, 356, 123-138. https://doi.org/10.3354/ meps 07230

León-Álvarez, D., Candelaria-Silva, C., Hernández-Almaraz, P. \& León-Tejera, H. (2007). Géneros de algas marinas tropicales de México: 1. Algas verdes. México: Facultad de Ciencias, UNAM. https://doi.org/10.22201/ fc.9786070289910e.2017
Littler, D. S. \& Littler, M. M. (2000). Caribbean reef plants: an identification guide to the reef plants of the Caribbean, Bahamas, Florida and Gulf of Mexico. EE. UU.: OffShore Graphics Inc.

Littlerr, D. S. \& Littler, M. M. (1997). An illustrated marine flora of the Pelican Cays, Belize. Proc. Biol. Soc. Wash., 9, 1-149.

Lot-Helgueras, A. (1971). Estudio de las fanerógamas marinas en las cercanías de Veracruz, Ver. An. Inst. Biol. Univ. Nac. Autón. Méx., Ser. Bot., 42(1), 1-48.

Luna-Ortega, I. \& De la Cruz-Francisco, V. (2017). Ensamblajes de macroalgas en superficies muertas de corales escleractinios (Anthozoa: Scleractinia) en el arrecife Oro Verde, Veracruz, México. CICIMAR Oceánides, 32(1), 11-23.

Martínez-Daranas, B., Esquivel, M., Alcolado, P. M. \& Jiménez, C. (2016). Composición específica y abundancia de macroalgas $\mathrm{y}$ angiospermas marinas entre arrecifes coralinos de la plataforma Sudoccidental de Cuba (1987). Hidrobiológica, 26(2), 323-337. https://doi.org/10.24275/uam/izt/dcbs/ hidro/2016v26n2/martinez

Martínez-Daranas, B., Macías-Reyes, D. \& Cano-Mallo, M. (2013). Protocolo para el monitoreo de los pastos marinos. Cuba: Centro Nacional de Áreas Protegidas.

Mateo-Cid, L. E., Mendoza-González, A. C. \& Galicia-García, C. (1996). Algas marinas de Isla Verde, Veracruz, México. Acta Bot. Mex., 36, 59-75. https://doi. org/10.21829/abm36.1996.762

Mateo-Cid, L. E., Mendoza-González, A. C., Ávila-Ortiz, A. G. \& Díaz-Martínez, S. (2013). Algas marinas bentónicas del litoral de Campeche, México. Acta Bot. Mex., 104, 53-92. https://doi. org/10.21829/abm104.2013.57 
Mendoza-González, A. C., Mateo-Cid, L. E., García-López, D. Y. \& Acosta-Calderon, J. A. (2014). Diversity and distribution of articulated Coralline algae (Rhodophyta, Corallinales) of the Atlantic coast of Mexico. Phytotaxa, 190(1), 045-063. https://doi. org/10.11646/phytotaxa.190.1.6

Moura, C. W. D. N., Almeida, W. R. D., Santos, A. A. D., Junior, A., Alves, A. M. \& Moniz-Brito, K. L. (2014). Polyphysaceae (Dasycladales, Chlorophyta) in Todos os Santos Bay, Bahia, Brazil. Acta Bot. Brasil., 28(2), 147-164. https://doi.org/10.1590/ s0102-33062014000200002

Ortega, M. M., Godínez, J. L. \& Garduño-Solórzano, G. (2001). Catálogo de algas bénticas de las costas mexicanas del Golfo de México y Mar Caribe. México: Instituto de Biología, Universidad Nacional Autónoma de México.

Ortiz-Lozano, L., Colmenares-Campos, C. \& Gutiérrez-Velázquez, A. L. (2019). Arrecifes Sumergidos y su Relevancia para el Corredor Arrecifal del Suroeste del Golfo de México. En: A. Granados-Barba, L. Ortiz-Lozano, C. González-Gándara \& D. Salas-Monreal (Eds.), Estudios Cientificos en el Corredor Arrecifal del Suroeste del Golfo de México (pp. 1-36). México: Universidad Autónoma de Campeche, México. https://doi.org/10.26359/ epomex0319

Ortiz-Lozano, L., Pérez-España, H., Granados-Barba, A., González-Gándara, C., Gutiérrez-Velázquez, A. \& Martos, J. (2013). The Reef Corridor of the Southwest Gulf of Mexico: Challenges for its management and conservation. Ocean Coast. Manage., 86, 22-32. https://doi.org/10.1016/j. ocecoaman.2013.10.006
Pacheco-Cervera, M. C., Pacheco-Ruíz, I., Ramos-Miranda, J., Cetz-Navarro, N. P. \& Soto-Ávila, J. L. (2010). Presencia del género Caulerpa en la Bahía de Campeche, Camp. Hidrobiológica, 20(1), 57-69.

Pedroche, F. F. \& Sentíes, A. (2003). Ficología marina mexicana. Diversidad y Problemática actual. Hidrobiológica, 13, 23-32.

Ramírez-García, P., Terrados M., J., Hernández Martínez, O., Pedraza, K. \& Quiroz, A. (2007). La vegetación de Thalassia testudinum en los arrecifes de Hornos, Sacrificios y Enmedio: Biomasa, productividad y dinámica de crecimiento. En A. Granados Barba, L. G. Abarca Arenas \& J. M. Vargas Hernández (Eds.), Investigaciones Cientificas en el Sistema Arrecifal Veracruzano (pp. 173-184). México: Universidad Autónoma de Campeche.

Ramírez-Rodríguez, A. \& Blanco-Pérez, R. (2011). Macroalgas bentónicas marinas: conocimiento actual. En Comisión Nacional para el Conocimiento y Uso de la Biodiversidad (Conabio) (Eds.), La biodiversidad en Veracruz, Estudio de Estado (pp. 51-57). México: Conabio/Gobierno del Estado de Veracruz/ Universidad Veracruzana/Instituto de Ecología A. C.

Robinson, N. M., Galicia-García, C. \& Okolodkov, Y. (2012). New records of Green (Chlorophyta) and Brown algae (Phaeophyceae) for Cabezo reef, National Park Sistema Arrecifal Veracruzano, Gulf of Mexico. Acta Bot. Mex., 101, 11-48. https://doi.org/10.21829/ abm101.2012.24

Romeu, E. (1996). Pastos marinos, una cuna para la biodiversidad. CONABIO. Biodiversitas, 5, 10-14.

Sentíes, A. \& Fujii, M. T. (2002). El complejo Laurencia (Rhodomelaceae, 
Rhodophyta) en el Caribe mexicano. En A. Sentíes \& K. M. Dreckmann (Eds.), Monografias ficológicas (pp. 119-192). México: UAM Iztapalapa y Red Latinoamericana de Botánica.

Solé, M. A. \& Foldats, E. (2003). El género Dictyota (Phaeophyceae, Dictyotales) en el caribe venezolano. Acta Bot. Venez., 26(1), 41-81.

Suárez, A.M., Martínez-Daranas, B. \& Alfonso, Y. (2015). Macroalgas marinas de Cuba. Cuba: Editorial Universidad de La Habana.

van Tussenbroek, B. I., Barba, M. G., Wong, J. G. R., van Dijk, J. K. \& Waycott, M. (2010). Guía de los pastos marinos tropicales del Atlántico oeste. México: Instituto de Ciencias del Mar y Limnología. https://doi.org/10.18475/cjos. v46i2.a26
Vieira, C., Camacho, O., Wynne, M.J., Mattio, L., Anderson, R. J., Bolton, J.J., Sansón, M., D'hondt, S., Leliaert, F., Fredericq, S., Payri, C. \& De Clerck, O. (2016). Shedding new ligth on old algae: Matching names and sequences in the Brown algal genus Lobophora (Dictyotales, Phephyceae). Taxon, 65, 689707. https://doi.org/10.12705/654.1

Zúñiga-Ríos, D., Martínez-Daranas, B. \& Alcolado, P. M. (2012). Ficoflora de los arrecifes coralinos del archipiélago Sabana-Camagüey, Cuba. Ser. Oceanol., 11, 57-76. 
